\title{
Witterungsänderungen in der Schweiz im 19. und 20. Jahrhundert Ursachen und Folgen
}

\section{Gletscherhochstände und Gletscherschwund in den letzten zwei Jahrhunderten}

Der drastische Gletscherschwund seit der Mitte des 19. Jahrhunderts bis in die heutige Zeit, wie er durch die beiden Bilddokumente anschaulich illustriert wird, ist uns nicht unbekannt, werden wir doch durch die gewaltigen Moränenwälle in den Gletschervorfeldern unserer großen vereisten Gebirgszonen bei jeder Alpenwanderung daran erinnert. Vom Beginn des 17. Jahrhunderts bis gegen 1850 lag die Stirnregion unserer Gletscher allgemein viel tiefer als heute, ja sie befand sich in einzelnen Zonen, wie z. B. in Grindelwald und im Montblancgebiet, in unmittelbarer Nähe der bewohnten Orte (vgl. ZUMBÜHL u. MESSERLI, 1980, sowie Fig. 1 und 2, HOLZHAUSER, 1984).

Auf was ist diese Tatsache zurückzuführen? Wir wissen, daß vor allem zwei Faktoren eine wesentliche Rolle im Gletscherhaushalt spielen: die Niederschläge als Spender in den hochgelegenen Gebieten mit hohem Schneeanteil und die sommerliche Temperatur als Verbraucher. Die Gletscher reagieren auf die Veränderung der beiden Parameter in ähnlicher Größenordnung (Lang, 1973). Die beiden Wetterelemente sind nicht unabhängig voneinander: kühle, sonnenarme Sommer bringen meist einen erhöhten Schneeanteil. Es stellt sich die Frage, ob Vorstoß und Rückgang der Gletscher, besonders in der Zeit von 1600 bis 1850, der sogenannten «Kleinen Eiszeit», auf Änderungen eines der beiden Elemente zurückzuführen ist und ob eventuell noch weitere Faktoren im Spiel sind.

Die Schwierigkeit, diese Frage zu beantworten, liegt in der Kürze der verfügbaren Meßreihen für die Temperatur und noch ausgeprägter für die Niederschlagsmengen. Aus der 2. Hälfte des 18. Jahrhunderts sind zwar einzelne Serien bekannt, doch kann bei den meisten Stationen für die Niederschläge nur die Häufigkeit des Auftretens, nicht aber die Menge aus den Aufzeichnungen direkt ermittelt werden. Wir brauchen nicht näher auf diese Grundlagen einzugehen, da O.GISLER diese Verhältnisse zu Ende des 18. Jahrhunderts eingehend in Heft 4/85 der Geographica Helvetica dargelegt hat. Es zeigt sich, daß es vom Jahr 1781 an möglich ist, die einzelnen Wetterlagen im europäischen Raum zu verfolgen und damit festzustellen, was sich im großräumigen Wettergeschehen seit diesem Zeitpunkt verändert hat. Natürlich müssen sie für das Jahrzehnt 1781-1790, für welches ausgiebiges Datenmaterial zur Verfügung steht, nicht unbedingt repräsentativ sein für den ganzen Zeitraum von 1600-1850, in welchem es immerhin einige größere Gletscherschwankungen gab, doch besteht die Möglichkeit, die Untersuchungen vom Startpunkt 1781 an bis in unsere Zeit, also auf mehr als einen Viertel der Dauer der «Kleinen Eiszeib», auszudehnen. Als Ausgangspunkt können die vielen bahnbrechenden Arbeiten von LAMB in England in den vergangenen drei Jahrzehnten dienen (LAMB, 1966, 1977). Es muß aber immer noch sehr viel Arbeit geleistet werden, handelt es sich doch bei der ersten Hälfte des 19. Jahrhunderts um eine meteorologisch sehr interessante, jedoch durch die Wirren des politischen Umbruchs im Gefolge der Französischen Revolution sehr datenarme Zeit mit daher erschwerten Auswertebedingungen. Wir kennen aus der Gletschergeschichte die beiden großen Vorstoßperioden vor 1820 und gegen die Jahrhundertmitte, wobei besonders der rasch einsetzende Hochstand von 1820 in bezug auf die Wetterlagenhäufigkeit wesentliche Einblicke in die Ursachen der Vorstöße verspricht.

Mit unseren heutigen Kenntnissen müssen wir annehmen, daß die Niederschlagsmengen bei den Gletscherhochständen sicher mitbeteiligt sind. Meist stellt jedoch der Temperatureinfluß das maßgebende Element dar durch die doppelte Wirkung der kühlen Sommer: Verminderung der Ablation und Erhöhung des Schneeanteils bei den Niederschlägen. Der Temperaturverlauf seit dem 18. Jahrhundert wurde bereits im Heft 1985/4 bei der Arbeit GISLER in Fig. 15 an Hand mehrerer Reihen dargestellt. In Ergänzung dazu sind hier in Fig. 3 am Beispiel der ältesten Bergstation Gr. St. Bernhard (2472 m) für die Zeit seit 1818 nochmals generell die Veränderungen in den Höhenlagen der Gletscherzungen dargestellt. Dabei zeigt die kürzere Säntisreihe $(2500 \mathrm{~m})$ ein ähnliches Bild. Die in Heft 1985/4 wiedergegebene Station Hohenpeißenberg in Bayern (989 m) weist ein vom schweizerischen Netz abweichendes Verhalten auf. Sie ist nicht in Fig. 3 aufgenommen, da vom Ende des

Max Schüepp, Prof., Dr., Bürglistr. 16, 8304 Wallisellen Gian Gensler, Prof., Dr., Schweiz. Meteorolog. Anstalt, Krähbühlstr. 58, 8044 Zürich 

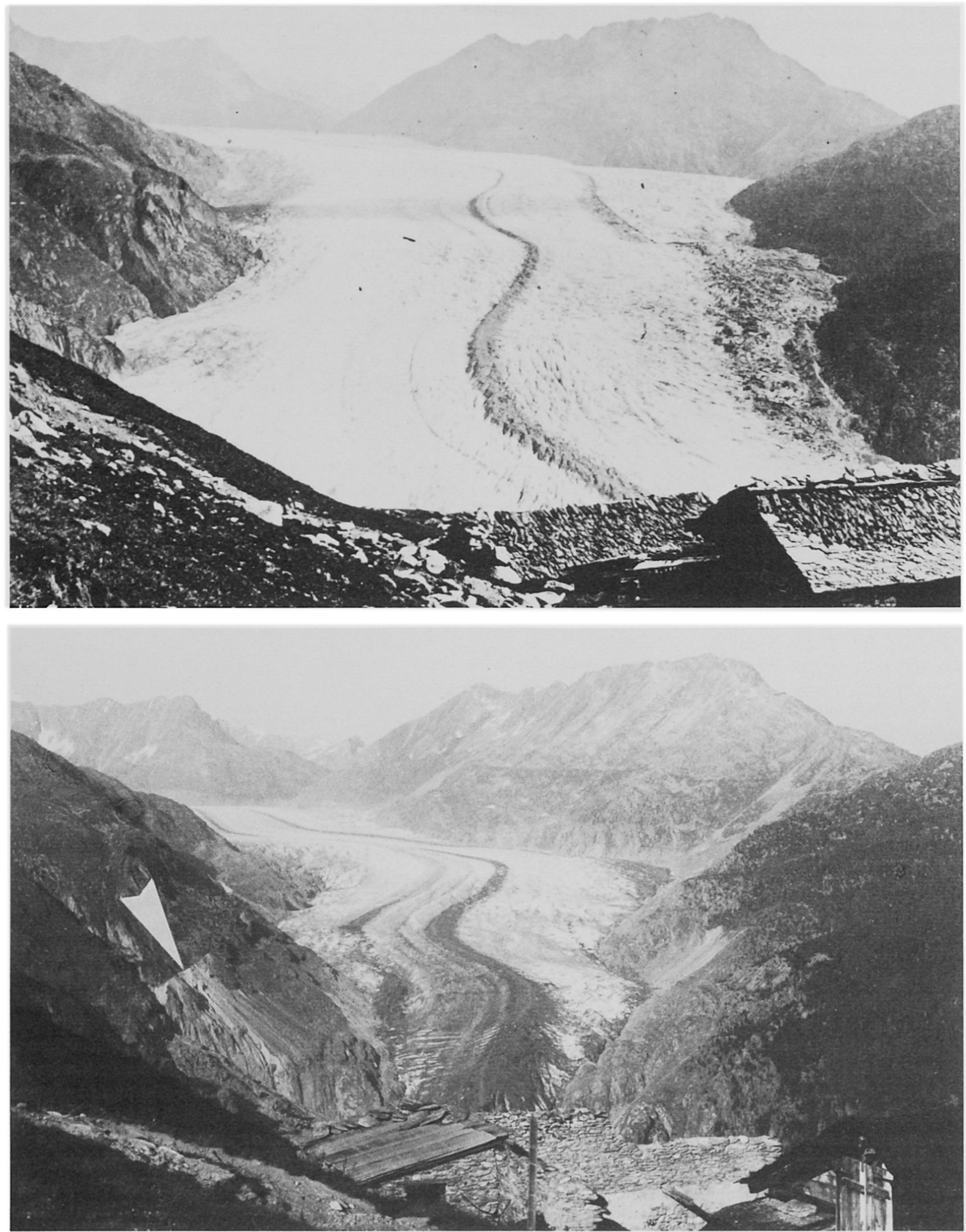

Fig. 1 und 2: Gletscherschwund in den Alpen, veranschaulicht am Beispiel des Großen Aletschgletschers (aus Holzhauser, 1984).

Fig. 1 (oben): Der Große Aletschgletscher von der Belalp aus gesehen im Jahre 1865 (Aufnahme Brun, Nr. 747, Archiv der Gletscherkommission).

Fig. 2 (unten): Vergleichphoto aus dem Jahre 1980. An der rechten Talseite ist der ehemalige Eisrand in der Nähe der «Tälli» an der anerodierten Ufermoräne (Pfeil) gut ersichtlich. 


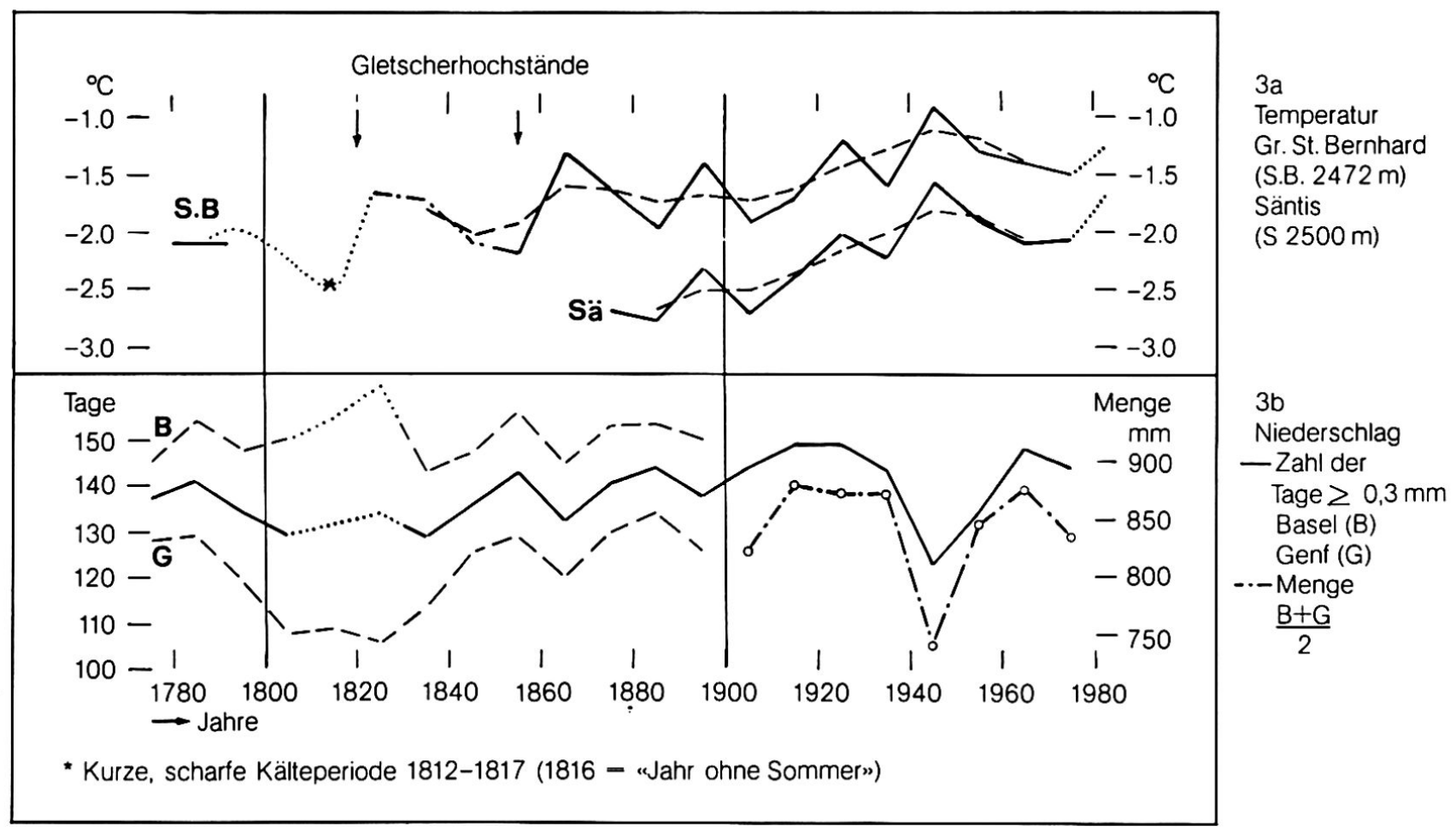

Fig. 3a Temperaturverlauf im 19. und 20. Jahrhundert in der Schweiz im Niveau von $2500 \mathrm{~m}$.

Bei der Temperatur verbindet die ausgezogene Linie die Jahrzehntenmittel. Der strichpunktierte Teil vor 1850 deutet an, daß die Messungen auf dem Gr. St. Bernhard im ersten Teil des 19. Jahrhunderts im Absolutbetrag etwas unsicher sind, da nicht genau bekannt ist, wie früh sich ein 1883 festgestellter Fehler von $+1 / 2^{\circ} \mathrm{C}$ beim jahrzehntelang verwendeten Thermometer entwickelt hatte. Das Mittel 1781-1792 wurde aus den auf den Gr. St. Bernhard reduzierten Beobachtungen des St. Gotthards berechnet. Bei der Säntisreihe ist eher die neueste Entwicklung unsicher, da mit der Einführung der automatischen Meßstation (1978) und der vorangehenden Bauperiode Standortverlegungen notwendig wurden, deren Auswirkungen sich erst provisorisch abschätzen lassen. Die gestrichelten Kurvenzüge geben den ausgeglichenen Verlauf nach der Formel 2 mal Jahrzehnt + vorangehendes Jahrzehnt + nachfolgendes Jahrzehnt / 4

Fig. 3b Niederschlagsverlauf.

Beim Niederschlag ist die Zahl der Tage mit Mengen von mindestens $0,3 \mathrm{~mm}$ für Basel und Genf angenähert bekannt, wobei jedoch im 19. Jahrhundert diese Größe von der Gewissenhaftigkeit des Beobachters abhängig ist. Der ausgezogene Mittelwert beider Stationen zeigt im Jahrzehnt 1941-1950 eine markante Senke, welche sich auch bei den gut mit der Zahl der Niederschlagstage korrelierten (strichpunktierten) Mengen findet. Für die Mengen ist ab 1960 erst eine provisorische Bearbeitung vorhanden infolge von Stationsverlegungen und Umstellung auf automatische Stationen.

Auch für die punktierte Periode 1801-1830 sind noch weitere Studien notwendig. Basel fiel von 1805-1825 aus und mußte durch Interpolationen mit Hilfe der Bearbeitungen von Schaffhausen durch Gisler (1983) vervollständigt werden. Dabei ergaben sich 1821-1830 bei Basel Maximalwerte, bei Genf dagegen gleichzeitig Minimalwerte, während in der übrigen Zeit die beiden Stationen gleichläufigen Gang aufweisen.

18. Jahrhunderts bis zum Jahr 1812 dort mit sehr großer Wahrscheinlichkeit infolge Veränderungen des Thermometernullpunktes etwas zu hohe Temperaturen gemessen wurden. Sie ist zudem für den Donauraum, nicht für die schweizerischen Verhältnisse repräsentativ. Die Gebiete östlich der Schweiz zeigen nach LAUSCHER (1980) ein eigenständiges Verhalten in jener Zeit, eine "Wärmeinsel» in einer kühleren Umgebung, deren Ursache, wenn sie sich im Rahmen der europäischen Gesamtprüfung als reell erweist, noch näher untersucht werden sollte.
In Fig. 3 sind auch die Niederschläge des 19. und 20. Jahrhunderts aufgenommen, wobei aber nur die Zahl der Niederschlagstage aus den beiden Talstationen Basel und Genf verwendet werden konnten, da aus den Höhenstationen keine langen homogenen Reihen vorliegen. Auch bei den Talstationen sind die Ergebnisse nur vorläufig, da besonders in der ersten Hälfte des 19. Jahrhunderts, wie GISLER (1983) feststellte, noch bedeutende Unterschiede zwischen den einzelnen Reihen bestehen (vgl. Erläuterungen zu Fig.3). Immerhin dürfte der allgemeine Verlauf 
gesichert sein, auf alle Fälle seit der Errichtung des schweizerischen allgemeinen Beobachtungsnetzes im Jahre 1863/64.

Werfen wir einen Blick auf den Kurvenverlauf von Temperatur und Niederschlag. Die Temperatur zeigt im 19. Jahrhundert ziemlich starke Ausschläge, ein Minimum vor dem 1855er Gletscherhochstand, gesamthaft jedoch vor 1820 bis gegen Ende des Jahrhunderts nur eine geringe Erwärmung. Dann finden wir aber einen markanten Anstieg im Jahresmittel, etwas verspätet in den Sommermonaten, wo sich die Temperaturzunahme erst nach einem kurzen teilweisen Gletschervorstoß ungefähr ab 1920 durchsetzt. Seit 1950 ist im Gebirge wieder leichte Abkühlung eingetreten. Die Messungen der letzten Jahre deuten auf eine Beendigung der Abkühlungsphase hin.

Bei der Niederschlagsmenge, welche sowohl zeitlich als auch örtlich selbst im Jahresmittel noch bedeutende Schwankungen aufweist, muß ein ausgleichender Mittelwert eingesetzt werden, wie ihn z.B. Uttinger bei der Bearbeitung des Blattes 12 des "Atlas der Schweiz" in den 1960er Jahren verwendet hat (UTTINGER, 1967). Im neuen, von W. Kirchhofer herausgegebenen Klimaatlas der Schweiz hat $\mathrm{Ch}$. Pfister in der zweiten Lieferung (1984) im Rahmen einer Gesamtbearbeitung seit 1525 ebenfalls ein schweizerisches Mittel berechnet, das einen ähnlichen Verlauf aufweist, sich aber vor allem auf die Verhältnisse in den Niederungen abstützt (PFISTER, 1984b). Fig. 4 gibt einen Ausschnitt aus den Pfisterschen Tafeln, welche sowohl Kurven für die Niederschläge als auch für die Temperatur aufweisen, wobei die Jahreszeiten getrennt aufgeführt werden. Es zeigen sich drei bedeutende Minima bei den Niederschlägen: ein erstes in den 1820er Jahren, entsprechend der Genfer Kurve in Fig. 3: ein zweites um 1870, welches vor allem im Sommer in Erscheinung tritt; und als drittes die große Trockenheit kurz vor der Mitte unseres Jahrhunderts, welche noch in der Erinnerung der älteren Generation fortlebt. Dabei zeigt sich im Vergleich der Temperatur- und Niederschlagskurven eindrücklich die Gegenläufigkeit, welche in vielen Perioden festzustellen ist, besonders in den Sommermonaten.

Wenn wir Fig. 3 und 4 in bezug auf den Gletscherhaushalt betrachten, sehen wir, daß dem Hochstand von 1820 ungewöhnlich tiefe Jahrestemperaturen vorausgingen, welche sowohl Sommer als auch Herbst und Winter umfassen. Die Niederschläge blieben dagegen mindestens in der Westschweiz unternormal, so daß die Temperatur offenbar maßgebend war für den Vorstoß. Auch 1850 war dies, wenn auch weniger ausgeprägt, der Fall. In der seit 1893 vorhandenen Gletschergeschichte (Fig. 5) zeigt sich der kleinere Vorsto $\beta$ vor 1920 , der auf sowohl kalte als auch niederschlagsreiche Sommer zurückzuführen ist. Seither war erst wieder im vergangenen Jahrzehnt, nach einem Rückgang der Temperaturen seit 1950 und zusätzlich höheren Winter- und Frühlingsnieder- schlagsmengen in den Jahren 1965-1970, ein Übergang zu erneutem Gletscherwachstum festzustellen. Hingegen brachte die Nachkriegszeit von 1945-1950 ein rapides Rückschmelzen der Eismassen im Gebirge, wobei die Gletscher mit tiefgelegenem Einzugsgebiet ihr Nährgebiet vorübergehend völlig einbüßten. Damit tritt in den letzten zwei Jahrhunderten die Sommertemperatur als maßgebende Einflußgröße für die Gletscherstände in Erscheinung. Wir müssen uns jedoch vor Verallgemeinerungen hüten. PFISTER kommt auf Grund seiner Bearbeitungen (1984b) zum Schluß, daß die gewaltigen Vorstöße der Gletscher in der 2. Hälfte des 16. Jahrhunderts vor allem durch ungewöhnlich hohe sommerliche Niederschläge verursacht wurden, allerdings gekoppelt mit unternormalen Temperaturen sowohl im Sommer als auch im Jahresmittel. Zudem ist anzunehmen, daß die Sonneneinstrahlung damals durch starke Bewölkung in der warmen Jahreszeit vermindert war.

\section{Die Ursachen der Klimaänderungen seit dem Ende des 18. Jahrhunderts}

GISLER hat (1985) die Witterungsverhältnisse des ausgehenden 18. Jahrhunderts untersucht. Hauptergebnisse: verlängerter, kalter Winter; verkürzte Übergangsjahreszeiten, besonders im Frühjahr; verhältnismäßig warme Sommer. Diese gegenüber heute verstärkte Kontinentalität ging nach LAMB (1977) mit einer großen winterlichen Eishäufigkeit im isländischen Raum einher und ist nach den PFISTERschen Untersuchungen charakteristisch für das 18. Jahrhundert. Warum hat seither ein Wechsel stattgefunden, der sich in einer Erhöhung der Wintertemperaturen, später auch in einer sommerlichen Erwärmung manifestierte? Noch ist es verfrüht, auf diese Frage eine endgültige Antwort zu geben, doch gehen wir, wie die Untersuchungen von SCHÖNWIESE $(1983,1984)$ zeigen, kaum fehl, wenn wir einen großen Teil der veränderten Verhältnisse auf das Konto der anthropogenen Einflüße buchen, daneben aber wohl auch auf Änderungen der Sonnenstrahlung, wenn wir den langjährigen Verlauf der Sonnenfleckenrelativzahlen betrachten (Fig. 6). Wir finden zu Beginn der Beobachtungsreihen um 1700 Minimalwerte (Ende des sogenannten Maunder-Minimums 1640-1715). Aber auch von 1800-1820, vor dem Gletscherhochstand, wurden ungewöhnlich tiefe Werte verzeichnet, während die höchsten Maxima kurz nach dem Temperaturmaximum in der Mitte des 20. Jahrhunderts auftraten. Diese Tatsachen deuten auf einen Zusammenhang, der in den Einzelheiten noch durch weitere Untersuchungen geklärt werden muß.

Auch bei den anthropogenen Einflüssen, bei welchen viele Faktoren zusammenspielen, stehen wir leider erst am Beginn der Abklärungen, obwohl dieses Gebiet von großer praktischer Bedeutung ist.

Das von Gisler bearbeitete Dezennium 1781-1790 stellt nur einen kleinen Ausschnitt aus dem durch 
Zehnjährige Mittel von Temperatur und Niederschlag in den Jahreszeiten.

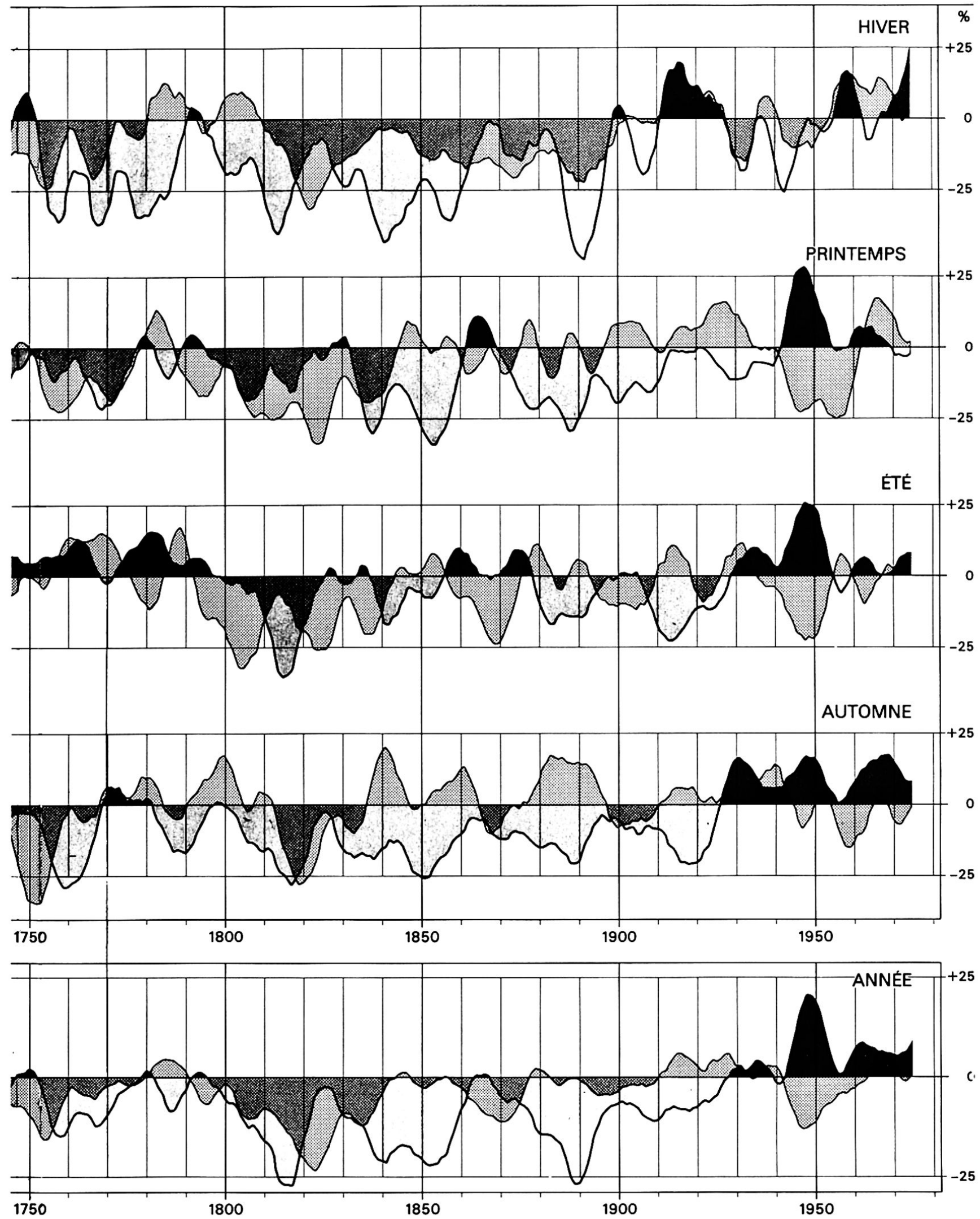

Fig. 4: Temperatur und Niederschlagsverlauf in den einzelnen Jahreszeiten und im Jahr nach Ch. Pfister (1984) auf Grund aller verfügbaren instrumentellen und anderen Unterlagen für die Niederungen der Alpennordseite. Die Schätzwerte für die Temperatur und den Niederschlag sind in Form gleitender gewichteter Mittel als Abweichung vom Mittel 1901-1960 dargestellt.

Dick ausgezogene Kurve = Temperatur

Dünn ausgezogene Kurve (mit Raster) = Niederschlag

Reproduziert mit Bewilligung des Bundesamtes für Landestopographie vom 4.3.86. 
Messungen belegten 200jährigen Zeitraum dar, welcher bereits 1966 durch Lamb und Johnson unter großem Arbeitsaufwand in Januar- und Juli-Wetterkarten bearbeitet wurde. Der detaillierte ganzjährige Überblick zeigt, da $\beta$ mit den extremen Monaten kein vollständiges Bild entsteht. Alle Monate, speziell die der Übergangsjahreszeiten, können eine wesentliche Rolle spielen. Es wäre daher wünschenswert, einen Gesamtüberblick des Zeitraumes seit 1781 zu erhalten. Dies gilt besonders für den Alpenraum, wo zur Interpretation der beiden Gletscherhochstände von 1820 und 1850 das Bedürfnis nach eingehender meteorologischer Bearbeitung besteht. Dabei ist, neben den bisher vorwiegend betrachteten Luftdruckund Temperaturverhältnissen, auch der Niederschlag eingehend $\mathrm{zu}$ bearbeiten, ferner die Wind- und Bewölkungsverhältnisse. Da für die Zeit vor 1935 keine Höhenwetterkarten bestehen, müssen die Strömungsverhältnisse indirekt erschlossen werden, was über witterungsklimatologische Einteilungen möglich ist. Die Bearbeitung von FLIRI (1984) für das zentrale Alpengebiet gibt die heutigen Verhältnisse wieder. Die Zeit vor 1935 war im Verhältnis Boden-Höhe nicht wesentlich verschieden, jedoch variierten die Häufigkeiten der einzelnen Witterungslagen, wie z.B. im März 1785 und Dezember 1788, im Vergleich zum heutigen Normalzustand (GISLER 1985).

Die Arbeiten für eine zwei Jahrhunderte umfassende Gesamtklimatologie sind daher sehr umfangreich, mühsam und nur mit zwischenstaatlicher Hilfe in der Bereitstellung allen verfügbaren Materials möglich. Einzelne übernationale Zentren in Europa müssen die Hauptlast tragen, z. B. das bereits von LAMB in Norwich gegründete Institut, welches sich speziell für die maritimen Belange einsetzen kann. Daneben wäre aber auch ein alpines Zentrum notwendig. Dafür steht gutes Material zur Verfügung in den Reihen Genf, St. Bernhard, Basel, Hohenpeißenberg, Wien und auf der Alpensüdseite Mailand neben all den vielen kürzeren Hilfsreihen wie Bern, Schaffhausen, Zürich usw.

Nun stellt sich die Frage: Ist dieser große Zeit- und Geldaufwand gerechtfertigt für die Untersuchung der Vergangenheit - sollte er nicht für andere Aufgaben eingesetzt werden? Wir bejahen das Bedürfnis, weil wir der Überzeugung sind, daß es für die Bewältigung der Zukunft notwendig ist, die vergangene Entwicklung genau zu kennen. Bis zur Mitte des 19. Jahrhunderts hatte der Mensch keinen wesentlichen Einfluß auf die klimatischen Bedingungen, abgesehen von lokalen Beeinflussungen z.B. durch Meliorationen oder andere Änderungen in der Bewirtschaftung. Bis in die 1880er Jahre waren die Einflüsse immer noch gering, obwohl die Industrie sich allmählich entwikkelte und damit die Luftverschmutzung einsetzte. Seither verschlechterten sich die Sichtverhältnisse in verschiedenen Gebieten.

Die in Zürich beobachtete Sichtverschlechterung (vgl. Fig. 14 bei Gisler, Geographica Helvetica
Nr.4/85, S. 220) ist keine lokale Erscheinung, wir finden sie allgemein beidseits der Alpen (RösLI, 1973; MÜLLER, 1980). Der Gang der Häufigkeitsabnahme der Alpensicht von Zürich aus für die Zeit von 1880 bis 1980 gibt einen Hinweis auf die bedeutende Transparenzabnahme in den unteren Schichten der Atmosphäre. Das Ergebnis der Arbeit von Graf (1984) überrascht insofern, als sich die Tage mit Alpensicht von Zürich aus wohl in ihrer Anzahl verringerten, jedoch nicht regelmäßig, sondern mit einem Sichtbarkeitsminimum kurz vor und während des Zweiten Weltkrieges. Sichtnotierungen durch die Schweizerische Meteorologische Anstalt in Zürich in diesen Jahren lassen erkennen, daß damals vorübergehend dichte Nebel mit Sichtweiten von nur 10 bis 20 Metern am Zürichbergabhang auftraten.

Nebst der Tatsache, daß bis 1945 noch nicht die saubere Ölheizung, sondern Holz und vor allem Kohle als Wärmeerzeuger dominierte, wurden zu jenen Krisen- und Kriegszeiten alle mehr oder weniger geeigneten Materialien zur zusätzlichen Wärmeerzeugung verwendet. Entsprechend hoch waren die Rauch- und Rußemissionen.

Der rapide Anstieg der Ölheizungsinstallationen nach 1945 hat zu einer wesentlichen Abnahme der Immissionsbelastung geführt. Hingegen führten die rasante Zunahme des Straßen- und Luftverkehrs und Erweiterungen im Industrie- und Dienstleistungssektor (z. B. Kehrichtverbrennungsanlagen) ab etwa 1960 zu einer erneuten Verminderung der Durchsichtigkeit der Luft. Dieser Prozeß scheint nach dem Ölschock von 1973

Tabelle 1: Globalstrahlung an diversen Orten in verschiedenen Meßperioden

\begin{tabular}{|c|c|c|c|c|}
\hline Station & $\begin{array}{l}\text { Höhe } \\
\text { m } \\
\text { ü.M. }\end{array}$ & Periode & Quelle & $\begin{array}{l}\text { Global- } \\
\text { strah- } \\
\text { lung } \\
\mathrm{kWh} / \mathrm{m}^{2}\end{array}$ \\
\hline $\begin{array}{l}\text { Karlsruhe } \\
\text { Basel }\end{array}$ & $\begin{array}{l}130 \\
316\end{array}$ & $\begin{array}{l}1895-1930 \\
1981-1984\end{array}$ & $\begin{array}{l}\text { GÖTZ } \\
\text { ASTA }\end{array}$ & $\begin{array}{l}1090 \\
1077\end{array}$ \\
\hline $\begin{array}{l}\text { Locarno- } \\
\text { Monti }\end{array}$ & 380 & $\begin{array}{l}1939-1941 \\
1958-1971 \\
1981-1984\end{array}$ & $\begin{array}{l}\text { GÖTZ } \\
\text { VALKO } \\
\text { ASTA }\end{array}$ & $\begin{array}{l}1375 \\
1374 \\
1228\end{array}$ \\
\hline Kloten & 436 & $\begin{array}{l}1963-1972 \\
1981-1984\end{array}$ & $\begin{array}{l}\text { VALKO } \\
\text { ASTA }\end{array}$ & $\begin{array}{l}1116 \\
1101\end{array}$ \\
\hline Zürich & $\begin{array}{l}493 \\
556\end{array}$ & $\begin{array}{l}1939-1942 \\
1981-1984\end{array}$ & $\begin{array}{l}\text { GÖTZ } \\
\text { ASTA }\end{array}$ & $\begin{array}{l}1217 \\
1104\end{array}$ \\
\hline Davos & $\begin{array}{l}1560 \\
1590\end{array}$ & $\begin{array}{l}1939-1941 \\
1958-1970 \\
1981-1984\end{array}$ & $\begin{array}{l}\text { GÖTZ } \\
\text { VALKO } \\
\text { ASTA }\end{array}$ & $\begin{array}{l}1467 \\
1346 \\
1365\end{array}$ \\
\hline Arosa & 1870 & $1901-1930$ & GÖTZ & 1478 \\
\hline Weißfluhjoch & 2690 & $\begin{array}{l}1970-1978 \\
1981-1984\end{array}$ & $\begin{array}{l}\text { VALKO } \\
\text { ASTA }\end{array}$ & $\begin{array}{l}1408 \\
1444\end{array}$ \\
\hline
\end{tabular}

ASTA = Jahrestabellen der Automatischen Stationen der Schweiz. 


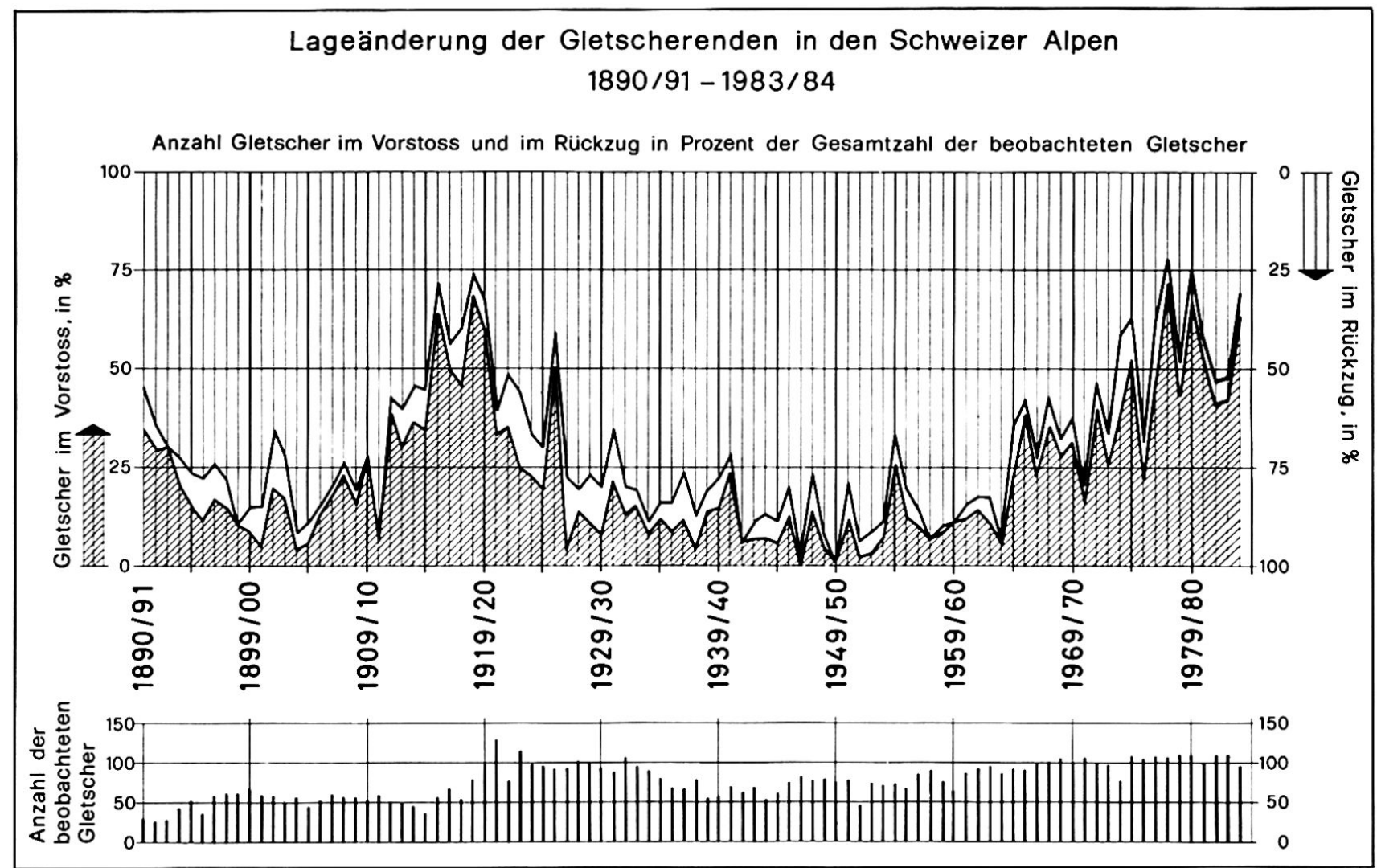

Fig. 5: Lageänderungen der Gletscherenden in der Schweiz seit 1890. Auszug aus dem 105. Bericht der Gletscherkommission der Schweizerischen Naturforschenden Gesellschaft, herausgegeben von der Versuchsanstalt für Wasserbau, Hydrologie und Glaziologie. Zeitschrift die Alpen, IV. Quartal, 1985, 61.Jahrgang, freundlicherweise zur Verfügung gestellt durch den Autor M. Aellen.

Sonnenflecken-Relativzahlen

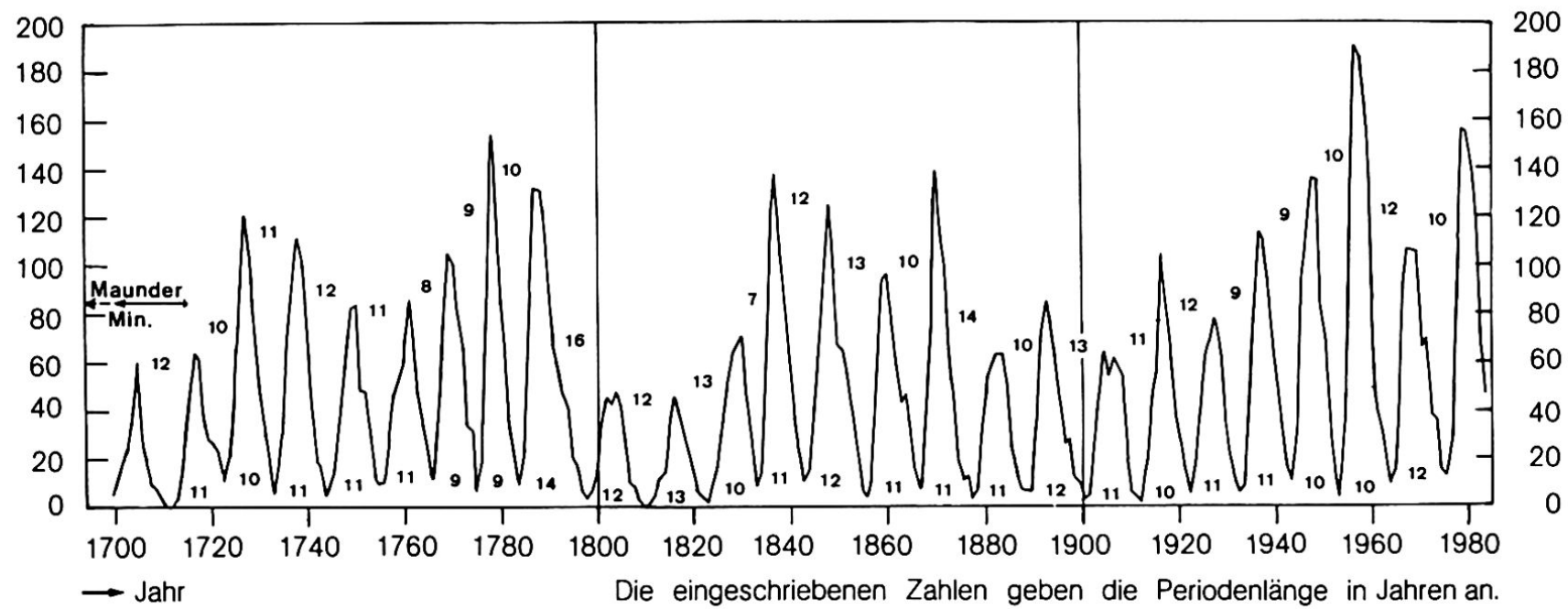

Fig. 6: Verlauf der Sonnenfleckenrelativzahlen nach Wolf seit 1700 auf Grund der freundlicherweise durch die "Specola solare ticinese" in Locarno-Monti zusammengestellten Reihe. Am linken Rand der Figur finden wir das Ende des "Maunder-Minimums" der Sonnenfleckentätigkeit, dann einen Anstieg bis 1780, von 1760-1780 mit "raschen Pulsschlägen», d. h. nur 9 Jahre Abstand zwischen den Maxima, dann den Abfall ab 1790 zu den tiefen Werten von 1800-1820, verbunden mit einer Verlangsamung del im Mittel 11jährigen Periode auf 12-15 Jahre und dem markanten Temperaturabfall der 1810er Jahre (Fig. 4!). Wir müssen annehmen, daß neben dem Vulkaneinfluß (Tambora-Ausbruch 1815) auch die Sonnenaktivität einen maßgebenden Einfluß auf die Wetterentwicklung hatte, auch wenn es heute noch nicht möglich ist, die Wirkungsweise der beiden Einflüsse genau zu trennen und statistisch sicher nachzuweisen. 
und zusätzlichen Bestrebungen zum Reinhalten der Luft seither gestoppt worden zu sein.

Ein weiterer Hinweis auf die heute "dickere Luft) können Vergleiche zwischen früheren und neuen Messungen der auf einer horizontalen Fläche auffallenden gesamten Strahlungsenergie geben. Diese aus der direkten Sonnenstrahlung und dem gestreuten Himmelslicht zusammengesetzte Globalstrahlung zeigt sinkende Tendenz, sofern eine korrekte Absoluteichung der Instrumente bereits für die ersten publizierten Messungen angenommen werden kann (GÖTZ, 1954; VALKO, 1980, vgl. Tab. 1).

Trotz der eingeschränkten Vergleichbarkeit ungleich langer Meßperioden läßt sich ein Energieverlust in der Größenordnung von 5 bis $10 \%$ im Laufe dieses Jahrhunderts erkennen. Eindrücklich ist, daß z. B. zu Beginn des Jahrhunderts der Strahlungsgenu $\beta$ in Arosa mindestens gleich hoch war wie in jüngster Zeit auf dem 800 Meter höher gelegenen Weißfluhjoch! Die Transparenzabnahme und die dadurch erhöhte Albedo der Atmosphäre hat somit nicht nur die Grundschicht beidseits der Alpen, sondern auch die freie Atmosphäre im und über dem Gebirge erfaßt.

Seit dem 2. Weltkrieg ist anstelle der Sichtabnahme durch Rauch die laufende chemische Verseuchung der Atmosphäre (SO2, NOx, $\mathrm{Pb}$ usw.) getreten, welche hier nicht näher erläutert werden soll. Sie wird in Zeiten des Waldsterbens bereits sehr eingehend andernorts diskutiert.

\section{Anstieg von Kohlendioxyd $\left(\mathrm{CO}_{2}\right)$ sowie weiterer sogenannter Treibhausgase (Distickstoffoxyd $\left(\mathrm{N}_{2} \mathrm{O}\right)$, Methan $\left(\mathrm{CH}_{4}\right)$, halogenisierter Kohlenwasserstoffe und Ozon als langfristig wirksamer Klimafaktor}

Die zur Zeit aktuelle Schadstoffdiskussion in Luft, Wasser und Boden bringt es mit sich, da $\beta$ die Aufmerksamkeit von einem wichtigen Klimafaktor abgelenkt wird, welcher für die langfristige Entwicklungsstrategie beim Übergang zum 21. Jahrhundert unbedingt berücksichtigt werden muß: das von uns in großen Mengen produzierte Kohlendioxyd und weitere, ähnlich wirkende Spurengase $\left(\mathrm{N}_{2} \mathrm{O}, \mathrm{CH}_{4}, \mathrm{O}_{3}\right.$ usw.), welche eine laufende Erwärmung bewirken.

In der Schweiz hat $\mathrm{H}$. Oeschger seit langem eindrücklich auf die Bedeutung des $\mathrm{CO}_{2}$-Anstieges für das atmosphärische Geschehen hingewiesen (Oeschger 1975, 1980; Neftel, Moor, Oeschger, Stauffer 1985). Schönwiese $(1983,1984)$ hat den $\mathrm{CO}_{2}$-Einfluß in den europäischen Klimareihen eindeutig festgestellt und kommt aufgrund der objektiven statistischen Analyse zum Schluß, daß ohne die von uns verursachte Zunahme der $\mathrm{CO}_{2}$-Konzentration in der Atmosphäre die mittlere hemisphärische Temperatur sich noch auf einem Stand befinden würde, welcher mit den Kälteperioden der «Kleinen Eiszeit», d.h. um 1810 oder 1610 , vergleichbar wäre.
Wir kennen Klimaschwankungen auch ohne menschliche Beeinflussung aus vorindustriellen Perioden: durch Änderungen der Sonnenstrahlung, große Vulkantätigkeit und gelegentliche Meteoritentreffer. Heute, im Zuge der weltweiten Vergrößerung des Wohlstandsgefälles auf der Erde zwischen den hochindustrialisierten Völkern und der sogenannten Dritten Welt, müssen wir versuchen, eine erhöhte Schwankungsbreite der Variationen und einen überlagerten allgemeinen Trend möglichst zu vermeiden (Flohn 1983, S. 203-204).

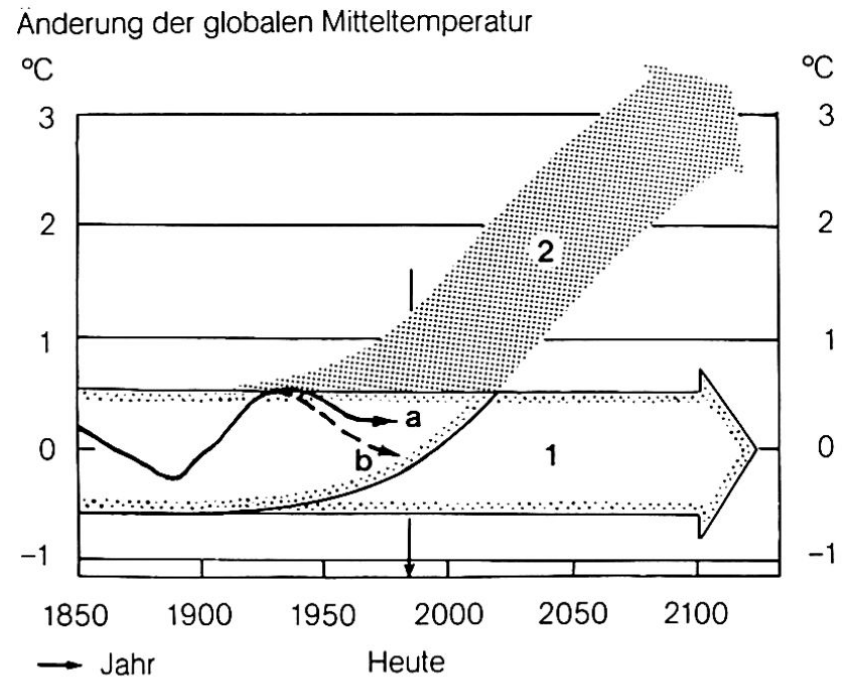

1) Bandbreite der natürlichen Schwankungen (klimatischer "Lärm")

2) Erwartete Bandbreite mit Einschluß des $\mathrm{CO}_{2}$-Effektes

a) Beobachtete bisherige Änderungen

b) Bisherige Anderungen ohne $\mathrm{CO}_{2}$-Effekt (?)

Fig. 7: Einfluß der "Treibhausgase" (vor allem $\mathrm{CO}_{2}$ ) auf den künftigen Temperaturverlauf in den gemäßigten Breiten. Nach Flohn und Fantechi, 1983, S. 191

Gleichzeitig stellt sich die Frage, ob wir es uns gestatten können, die in Hunderten von Millionen Jahren angesammelten Erdölvorräte im Laufe von wenigen Menschenaltern zu verbrauchen. Diese Unbekümmertheit steht im krassen Gegensatz zu der vielfach übergroßen Angst vor den Einflüssen der Strahlungsbelastung durch die Abfälle der Kernkraftwerke, welche keine vermehrte $\mathrm{CO}_{2}$-Belastung bringen, sondern im Gegenteil helfen, diese konstantzuhalten. Die durch den Namen Atom unwillkürlich hergestellte Verbindung zu den Atombomben, deren Verwendung die Schrecken eines heutigen Krieges noch ungemein verstärken würde, darf uns nicht darüber hinwegtäuschen, daß es die Atomenergie ist, welche sowohl vom Sonnenball als auch vom Erdinnern her das Leben auf der Erde im Gang hält. Wir sollen zwar bei den hochtechnisierten Völkern das Energiewachstum nicht ungezügelt vorantreiben, müssen es aber bei den Völkern der Dritten Welt stark 
anheben, wenn Gleichheit und Brüderlichkeit, unsere Ideale seit der Französischen Revolution, erfüllt werden sollen.

Damit kommen wir unweigerlich auf eine weiterhin starke Erhöhung des Energiekonsums für die gesamte Menschheit, den wir nicht mit fossilen Brennstoffen, d.h. mit einer erhöhten $\mathrm{CO}_{2}$-Produktion und den damit verbundenen Klimaveränderungen, bewältigen sollten, sondern nur in Verbindung von Kernenergie (in fernerer Zukunft wohl nicht nur durch Spaltung, sondern auch durch Fusion) sowie vermehrter Nutzung der primären Sonnenstrahlung speziell im subtropischen Gürtel und Windenergie sowie geothermischer Energie an geeigneten Punkten.

\section{Aktuelle und künftige Aufgaben der Klimaforschung}

Die Klimatologie hat die Aufgabe, das Material für die Entwicklung von Klimamodellen bereitzustellen, welche uns gestatten, die durch menschliche Eingriffe verursachten Veränderungen $\mathrm{zu}$ berechnen, damit rechtzeitig eventuelle Schäden erkannt und vermieden werden können (BERGER, NICOLIS, 1984). Wir dürfen uns bei den Beobachtungsnetzen nicht auf die traditionellen Wetterelemente beschränken, sondern müssen alle Komponenten, z. B. den $\mathrm{CO}_{2}$-Gehalt der Atmosphäre, mit einbeziehen. Ferner sollten die Strahlungsverhältnisse ebenfalls in Form von klimatologischen Bearbeitungen allgemein zugänglich gemacht werden. Ist es doch nur einem kleinen Bruchteil der Menschen bewußt, daß wir seit jeher einem ständigen Beschuß durch die kosmische Strahlung ausgesetzt sind, je nach Ort und Höhenlage mit bedeutenden Unterschieden. Dazu kommt vom Erdboden her eine Beeinflussung durch das radioaktive Radongas und seine Zerfallsprodukte, welches eine ähnliche Größenordnung wie die Bestrahlung von oben erreicht. Total mißt man bei uns in der für die Strahlenbelastung verwendeten Einheit Millirem (mrem) meist etwa 300-400 mrem pro Jahr (Huber, 1983), strichweise aber - speziell in größerer Meereshöhe - 2000 mrem. Die zusätzliche Belastung durch die künstlich erzeugte Radioaktivität beläuft sich direkt neben einem Kernkraftwerk auf wenige Milli$\mathrm{rem} / \mathrm{Jahr}$ und liegt sonst in der Schweiz unter $1 \mathrm{mrem}$, etwa soviel, wie wir von einem Fernsehapparat erhalten. Aus den medizinischen Behandlungen für unsere Gesundheit beziehen wir etwa 80 mrem pro Jahr. Diese Zahlen sollten uns klarmachen, daß die unsichtbaren Strahlenkräfte nur im Übermaß lebensfeindlich sind, und wir müssen uns davor hüten, die mittelalterlichen Hexen durch andere unbegründete Ängste zu ersetzen, sondern objektiv an die sich uns stellenden Probleme herangehen. Ist es nicht bezeichnend für unsere Zeit, daß die Kühltürme unserer Kernkraftwerke - etwas vom Ungefährlichsten, was es gibt! - für viele zum Symbol alles Bösen geworden sind? Warum haben so viele Men- schen Angst vor den kleinen Mengen von Abfällen unserer Reaktoren, deren Strahlung genau berechnet werden kann in alle Zukunft und welche zudem genau überprüft wird durch eine kompetente Fachkommission?

Die durch die moderne Technik eingesparten Arbeitskräfte müssen im Forschungsbereich eingesetzt werden, wo durch die ständige Entwicklung im Laufe der Millionen von Jahren sich auch immer neue Probleme stellen. Uns Menschen ist das Steuer in die Hand gegeben mit der Verantwortung gegenüber der gesamten Schöpfung. Wir sollen die Schwierigkeiten aber nicht ohne, sondern mit den heutigen technischen Mitteln meistern. Dabei dürfen wir nicht erst mit unseren Untersuchungen einsetzen, wenn ein Problem dringend wird wie z. B. bei der Schädigung unserer Wälder durch den übermäßigen Verbrauch an fossilen Energieträgern.

Wir haben am Anfang unserer Betrachtung die Gletscherschwankungen veranschaulicht, welche durch die relativ kleinen Temperatur- und Niederschlagsänderungen der letzten 200 Jahre verursacht wurden. Ein weltweiter Anstieg der Temperatur um $1,5-4,5^{\circ}$ und ein damit gekoppelter Anstieg des Meeresspiegels um 20-140 cm, wie er uns infolge der ständig zunehmenden Treibhausgase durch unseren Konsum der fossilen Brennstoffe im Laufe des nächsten Jahrhunderts bevorsteht, wird viel weiterreichende Konsequenzen haben (UNP/WMO/ICSU Konferenz Villach 1985). Dadurch wird die Zirkulation in der Lufthülle wesentlich verändert mit den entsprechenden notwendigen Umstellungen in der Weltwirtschaft, nicht nur in den Randgebieten wie z. B. in der Sahelzone.

Am Schlusse der instruktiven, allgemeinverständlichen 30seitigen Übersicht der Schweizerischen UNESCO-Kommission (AUF DER MAUR, 1983) schreibt Hans Oeschger zum Thema: «Wie wir unsere Erde zum Treibhaus machen» unter dem Titel: «Bleibt die Menschheit kurzsichtig?»: «Es ist heute noch nichts davon zu erkennen, daß sich das Handeln an einer längerfristigen Planung orientiert. Die Diskussionen um den Komplex Energie-Umwelt sind oft in Detailfragen erstarrt, doch nur die Sicht der Gesamtheit der Probleme und der kritische Vergleich der Möglichkeiten können zu optimalen Lösungen führen. Auch hat die Gesellschaft bisher immer erst auf Krisen reagiert, wenn sie spürbar aufgetreten sind. Im Falle der $\mathrm{CO}_{2}$-bedingten Erwärmung ist jedoch ein Handeln erforderlich, bevor die Krise einsetzt.» (HANS OESCHGER, Physikalisches Institut der Universität Bern, 1983). Speziell als Geographen haben wir die Aufgabe, die zu erwartenden Probleme rechtzeitig zu erkennen und mit Hilfe der Spezialisten ins allgemeine Bewußtsein zu bringen, damit geeignete $\mathrm{Maßnahmen} \mathrm{von} \mathrm{unseren} \mathrm{Behörden} \mathrm{geplant} \mathrm{und}$ geprüft werden können, aber auch vom ganzen Volk im Rahmen unserer demokratischen Verfassung getragen werden. 


\section{Literatur:}

AELLEN, M. (1985): Auszug aus dem 105. Bericht der Gletscherkommission der Schweizerischen Naturforschenden Gesellschaft, herausgegeben von der Versuchsanstalt für Wasserbau, Hydrologie und Glaziologie. In: Die Alpen IV. Quartal 1985, 61. Jahrgang, Zürich.

AUF DER MAUR, F. (1983): Wie wir unsere Erde zum Treibhaus machen. Nationale Schweizerische UNESCO-Kommission, Schweizerische Naturforschende Gesellschaft, 30 S., Bern.

BERGER, A., NICOLIS, C. (1984): New Perspectives in Climate Modelling, Developments in Atmospheric Sciences, Nr. 16.

FLIRI, F., SCHÜEPP, M. (1984): Synoptische Klimatographie der Alpen zwischen Mont Blanc und Hohen Tauern. In: Wissenschaftliche Alpenvereinshefte, Heft 29, Innsbruck.

FLOHN, H., FANTECHI, R. (1984): The climate of Europe, past, present and future. Natural and man-induced climatic changes: A European Perspective. D. Reidel Publishing Company, Dordrecht/Boston, Lancaster.

FLOHN H. (1985): Das Problem der Klimaänderungen in Vergangenheit und Zukunft. Erträge der Forschung Band 220 228 S, Wissenschaftliche Buchgesellschaft, Darmstadt.

GISLER, O. (1983): Die meteorologischen Beobachtungen von Schaffhausen (1794-1845) und Zürich (1767-1802) nebst einigen Betrachtungen über historische Niederschlagsreihen, Physische Geographie, Vol. 12, Geographisches Institut der Universität Zürich.

GISLER, O. (1985): Das Wetter zu Ende des 18. Jahrhunderts. In: Geographica Helvetica 4/1985, S. 205-222.

GÖTZ, F.W.P. (1954): Klima und Wetter in Arosa, Huber, Frauenfeld.

GRAF, A. (1984): Die Alpensicht von Zürich seit 1865 und ihre Abhängigkeit von Wetterlage, Tages- und Jahreszeit. Diplomarbeit ausgeführt am Geographischen Institut der Universität Zürich.

HOLZHAUSER, H.P. (1984): Zur Geschichte der Alpengletscher und des Fieschergletschers. Physische Geographie Uni Zürich, Vol. 13, 452 S., Zürich.

HUBER, O. (1984): 27. Bericht der Eidg. Kommission zur Überwachung der Radioaktivität für das Jahr 1983 zuhanden des Bundesrates, S. 1-2 u. 28-31, Fribourg

LAMB, H. H., (1977): Climate, Present, Past and Future, Vol. 2 , Climatic History and the Future, London, Methuen.

LAMB, H. H., JOHNSON, A.I. (1966): Secular variations of the atmospheric circulation since 1750, Geophysical Memoires, vol. XIV, No 110, Meteorological Office, HMSO, London.
LANG, H., DAVIDSON, G. (1973): Beitrag zum Problem der klimatischen Schneegrenze. Verhandlungen der Schweiz. Naturforsch. Gesellschaft, S. 158-160, Birkhäuser, Basel.

LAUSCHER, F. (1980): Die Wärmeinsel des österreichischen Donauraumes zur Regierungszeit Kaiser Franz Josephs II. In: Annalen der Meteorologie, Neue Folge, Nr.16, p.199/200, Offenbach.

MÜLLER, W. (1980): Nimmt die Lufttrübung zu? In: Archiv für Meteorologie, Geophysik und Bioklimatologie, Serie B, Heft 28 , p. 227-236, Springer, Wien.

NEFTEL, A., MOOR, E., OESCHGER, H., STAUFFER, B. (1985): Evidence from polar ice cores for the increase of atmospheric $\mathrm{CO}_{2}$ in the past two centuries. In: Nature, Vol. 315, S. 45-47.

OESCHGER, H., SIEGENTHALER, U., SCHOTTERER, U., GUGELMANN, A. (1975): A box diffusion model to study the carbon dioxide exchange in nature. Tellus 72/1975 (2): S. $168-192$.

OESCHGER, H., MESSERLI, B., SVILAR, M. (1980): Das Klima, Analysen und Modelle, Geschichte und Zukunft. Springer, Berlin.

PFISTER, CHR. (1984a): Klimageschichte der Schweiz 1525 bis 1860. Das Klima der Schweiz 1525 bis 1860 und seine Bedeutung in der Geschichte von Bevölkerung und Landwirtschaft, Band 1, Haupt, Bern.

PFISTER, CHR. (1984b): Zehnjährige Mittel von Temperatur und Niederschlag in den Jahreszeiten Blatt 14.4 in Kirchhofer, W. Klimaatlas der Schweiz 2. Lieferung. Verlag des Bundesamtes der Landestopographie Bern.

RÖSLI, H.P. (1973): Abnahme der horizontalen Sichtweite in der Magadinoebene. In: Verhandlungen der Schweizerischen Naturforschenden Gesellschaft 1973, p. 122-125.

SCHÖNWIESE, C.D. (1983, 1984): Northern Hemisphere Temperature Statistics and Forcing. Part A (1983) 1881-1980 AD. In: Archiv für Meteorologie, Geophysik und Bioklimatologie, Serie B, Vol.32 p. 337-360, Part B (1984) 1579-1980 AD, Vol. 35, p. 155-178, Springer, Wien

UTTINGER, H. (1967): Mittlere jährliche Niederschlagsmengen in cm 1901-1940 und Niederschlagshäufigkeit 1901-1960, Blatt 12. In: Imhof, E., Atlas der Schweiz., Eidg. Landestopographie, Bern.

VALKO, P. (1980): Strahlung, 2. Teil, Klimatologie der Schweiz, Heft 26/l, Beiheft zu den Annalen Jahrgang 1979 der Schweizerischen Meteorologischen Anstalt, Zürich.

ZUMBÜHL, H., MESSERLI, B. (1980): Gletscherschwankungen und Temperaturverlauf. Beispiel einer Korrelationsanalyse von indirekten und direkten Klimazeugen am Beispiel der Grindelwaldgletscher und der 210jährigen Basler Temperaturreihe. In: Oeschger, Messerli und Svilar: Das Klima S. 161-174, Springer, Berlin. 